\title{
Targeting cryptic epitope with modified antigen coupled to the surface of liposomes induces strong antitumor CD8 T-cell immune responses in vivo
}

\author{
YUTAKA HORIUCHI, AKIRA TAKAGI, TETSUYA UCHIDA and TOSHITAKA AKATSUKA \\ Department of Microbiology, Faculty of Medicine, Saitama Medical University, Moroyama-cho, \\ Iruma-gun, Saitama 350-0495, Japan
}

Received June 24, 2015; Accepted July 20, 2015

DOI: $10.3892 /$ or.2015.4299

\begin{abstract}
Active cancer immunotherapy, such as cancer vaccine, is based on the fundamental knowledge that tumor-associated antigens (TAAs) are presented on MHC molecules for recognition by specific $\mathrm{T}$ cells. However, most TAAs are self-antigens and are also expressed on normal tissues, including the thymus. This fact raises the issue of the tolerance of the TAA-specific T-cell repertoire and consequently the inability to trigger a strong and efficient antitumor immune response. In the present study, we used antigens chemically coupled to the surface of liposomes to target telomerase reverse transcriptase (TERT), a widely expressed self/tumor antigen. Taking advantage of the high homology between mouse and human TERT, we investigated immunogenicity and antitumor efficiency of the liposomal TERT peptides in HLA-A*0201 transgenic HHD mice. Using the heteroclitical peptide-modifying approach with antigen-coupled liposomes, we identified a novel cryptic epitope with low affinity for HLA*0201 molecules derived from TERT. The heteroclitical variant derived from this novel low affinity peptide exhibited strong affinity for HLA*0201 molecules. However, it induced only weak CD8 T-cell immune responses in HHD mice when emulsified in IFA. By contrast, when coupled to the surface of the liposomes, it induced powerful CD8 T-cell immune responses which cross-reacted against the original cryptic epitope. The induced CD8 T cells also recognized endogenously TERT-expressing tumor cells and inhibited their growth in HHD mice. These data suggest that heteroclitical antigen derived from low affinity epitope of
\end{abstract}

Correspondence to: Dr Yutaka Horiuchi, Department of Microbiology, Faculty of Medicine, Saitama Medical University, 38 Morohongo, Moroyama-cho, Iruma-gun, Saitama 350-0495, Japan E-mail: horiuchi@saitama-med.ac.jp

Key words: tumor vaccine, surface-linked liposomal antigen, heteroclitical peptide, cryptic epitope, telomerase reverse transcriptase, immune tolerance tumor antigens coupled to the surface of liposome may have a role as an effective cancer vaccine candidate.

\section{Introduction}

Tumor-associated antigen (TAAs)-specific T cells are detected in many patients with cancer $(1,2)$. However, these TAA-specific $\mathrm{T}$ cells typically fail to control tumor growth. It is expected that vaccination with TAA enhances tumor-specific $\mathrm{T}$ cell responses, improves antitumor immunity and provides clinical benefit. The development of cancer vaccines has been greatly potentiated by the availability of synthetic tumor-antigenpeptides, although progress has been hampered by the poor immunogenicity of antigen-peptides.

Liposome has been proposed to be a carrier of antigens and an adjuvant to induce immune responses (3). We previously reported that antigens chemically coupled to the surface of liposomes consisting of unsaturated fatty acids were cross-presented by antigen-presenting cells (APCs) to cytotoxic T lymphocytes (CTLs) (4). Moreover, we have shown that single immunization of as low as $280 \mathrm{ng}$ of liposomal peptide along with $\mathrm{CpG}$ conferred complete protection to the immunized mice from a highly virulent mutant strain, clone 13 of lymphocytic choriomeningitis virus (LCMV). In addition, surface-linked liposomal antigens induced long-lived memory $\mathrm{CD}^{+} \mathrm{T}$ cells in the absence of $\mathrm{CD} 4^{+} \mathrm{T}$-cell assistance $(5,6)$. This form of CTL-based liposomal vaccine was also investigated for tumor vaccine using an OVA-expressing mouse tumor model. This investigation suggested surface-coupled liposomal antigens may be applicable for the development of tumor vaccines (4). However, to the best of our knowledge, the possibility of a vaccine targeting endogenous tumor antigen by surface-coupled liposomal antigens remains to be examined.

Telomerase reverse transcriptase (TERT) is a tumor antigen that is highly expressed in $>85 \%$ of all human types of cancer including stem cell-like tumor cells (7-10). Therefore, TERT antigens have the potential to mediate antitumor immune responses against a range of tumors (11). In previous studies, epitope peptides derived from TERT have been shown to elicit CTL responses capable of recognizing TERT-expressing tumor cells in vitro $(7,8,12)$. However, reports of antitumor efficiency in vivo using natural TERT peptide are limited. 
Self-antigen peptides do not necessarily undergo antigen processing/presentation and serve as dominant epitopes. In certain situations, the peptides are hidden from immune recognition and termed 'cryptic' (13). Dominant peptides show a high affinity for MHC molecules and are frequently presented by somatic cells, whereas, cryptic peptides show a low affinity for MHC molecules and are infrequently presented by somatic cells. Similar to many other TAAs, TERT is a self-antigen. Therefore, it is thought that central tolerance against TERT-derived peptides with high affinity for MHC molecules exists. The central tolerance against TAAs is deemed to be a major barrier for TAA-specific antitumor immunotherapy. On the other hand, the CTL repertoire specific for cryptic epitope may escape from the negative selection process in central tolerance. Therefore, CTLs against cryptic epitopes may be available for antitumor immunotherapy. However, it is difficult to identify cryptic epitopes for immunotherapy due to their poor immunogenicity. To circumvent this problem, previous investigators have created modified heteroclitical peptides to enhance their affinity for HLA-A2 molecules while preserving the amino acids that interact with the T-cell receptor (TCR), which is specific to the original cryptic epitope (14-17). Previous findings showed that vaccination with heteroclitical peptide derived from cryptic epitopes of TERT inhibited the growth of tumors in vivo $(15,23)$.

The aim of the present study was to determine the possibility that the liposome coupled with a peptide derived from TERT may serve as an effective CTL-based vaccine against tumor. First, to identify the optimum peptide for coupling on the surface of liposomes, 20 TERT-derived peptides with HLA-A2.1 binding motif were selected by computer algorism, the liposome-coupled peptide was prepared and their ability to provoke peptide specific CD8 $\mathrm{T}$ cell responses in HLA-A*0201-transgenic (HHD) mice was determined. We identified a novel cryptic epitope with low affinity for HLA molecules and found that immunization using liposome-coupled heteroclitical peptide derived from this novel cryptic epitope elicits CD8 T-cell responses against native peptide and efficiently inhibits the growth of TERT expressing tumors in HHD mice. Our results strongly support the use of liposomal vaccine surface coupled with TERT antigens as a new potential option for active immunotherapy against tumors.

\section{Materials and methods}

Prediction of CTL epitopes. To define potential HLA-A*0201-binding peptides from human TERT (hTERT), a computer-based program, BIMAS (http:// www-bimas.cit. nih.gov/molbio/hla_bind/), was used. As shown by the odd numbers in Table I, 10 peptides that have superior Bimas scores and homologous sequence in murine TERT (mTERT) were selected for predicted CTL epitopes.

Modified heteroclitical peptides were produced to enhance their affinity for HLA-A2 molecules while preserving the amino acids that interact with the TCR as previously described (18-21). Heteroclitical modifications were performed by replacing the amino acid at position 1 with a tyrosine $(\mathrm{Y})$, position 2 with a leucine $(\mathrm{L})$ or position 9 with a valine $(\mathrm{V})$. Heteroclitical peptides are shown by the even numbers in Table I. These peptides were synthesized by Eurofins Genomics (Tokyo, Japan).
Animals. The HLA-A*0201-transgenic HHD mice (a kind gift from Dr F.A. Lemonnier, Pasteur Institute, Paris, France) expressed a transgenic HLA-A*0201 monochain in which human $\beta-2$ microglobulin $(\beta 2 \mathrm{~m})$ is covalently linked to a chimeric heavy chain composed of HLA-A*0201 ( $\alpha 1$ and $\alpha 2$, domains) and $\mathrm{H}-2 \mathrm{Db}(\alpha 3$, transmembrane and cytoplasmic domains) (18). Six- to 12-week-old mice were used for all the experiments. The mice were housed in appropriate animal care facilities at Saitama Medical University (Saitama, Japan) and handled according to international guidelines. Experimental protocols were approved by the Animal Research Committee of Saitama Medical University.

Measurement of peptide relative affinity (RA) for HLA-A2.1. $\mathrm{T} 2$ cells $\left(3 \times 10^{5} / \mathrm{ml}\right)$ were incubated with various concentrations of peptides ranging from 100 to $0.1 \mu \mathrm{M}$ in serum-free RPMI-1640 medium (R0) supplemented with $100 \mathrm{ng} / \mathrm{ml}$ human $\beta 2 \mathrm{~m}$ (Acris Antibodies, San Diego, CA, USA) at $37^{\circ} \mathrm{C}$ for $16 \mathrm{~h}$, as previously described (16). The cells were then stained with the HLA-A2.1 molecule-specific BB7.2 mAb (BioLegend, San Diego, CA, USA). For each peptide concentration, HLA-A2.1-specific staining was calculated as the percentage of the staining obtained with $100 \mu \mathrm{M}$ of the reference peptide HIVpol589 (IVGAETFYV). The RA was determined as: $\mathrm{RA}=$ concentration of each peptide that induces $20 \%$ of HLA-A2.1 expression/concentration of the reference peptide that induces $20 \%$ of HLA-A2.1 expression. Lower RA value shows higher binding affinity for HLA-A2.1.

Assessment of peptide/HLA-A2.1 complex stability. T2 cells were incubated with $100 \mu \mathrm{M}$ of each peptide in $\mathrm{R} 0$ supplemented with $100 \mathrm{ng} / \mathrm{ml}$ human $\beta 2 \mathrm{~m}$ at $37^{\circ} \mathrm{C}$ for $16 \mathrm{~h}$, as previously described (16). The cells were then washed four times to remove free peptides, incubated with $0.2 \%$ GoldiPlug (BD Biosciences Pharmingen, San Diego, CA, USA) in R0 for $1 \mathrm{~h}$ to block the cell surface expression of newly synthesized HLA-A2.1. After washing and incubation for $0,2,4,6$ or $8 \mathrm{~h}$, the cells were stained with anti-HLA-A2.1 antibody (BB7.2). For each time-point, the peptide-induced HLA-A2.1 expression was calculated as: mean fluorescence of peptide-preincubated T2 cells - mean fluorescence of T2 cells treated in similar conditions in the absence of peptide. $\mathrm{DC}_{50}$ (dissociation complex, DC) was defined as the time required for the loss of $50 \%$ of the HLA-A2.1/peptide complexes stabilized at $\mathrm{t}=0$.

Cell lines and telomerase detection. Mouse lymphoma cell lines RMA and RMA-HHD, which were a kind gift from Dr F.A. Lemonnier (22) were cultured in RPMI-1640 medium supplemented with $10 \%$ fetal bovine serum (FBS) (R-10). Telomerase detection in tumor cells was achieved by flow cytometry as previously reported using anti-hTERT monoclonal antibody (H-231; Santa Cruz Biotechnology, Inc., Dallas, TX, USA), which cross reacts with mTERT (23).

Liposome. The liposomes used in the present study were provided by NOF Corporation (Tokyo, Japan), and consisted of dioleoyl phosphatidylcholine (DOPC), dioleoyl phosphatidylethanolamine (DOPE), dioleoyl phosphatidyl glycerol (DOPG) and cholesterol in a 4:3:2:7 molar ratio. The crude 
Table I. Bimas score, HLA-A2.1 affinity and stabilization capacity of hTERT peptides.

\begin{tabular}{|c|c|c|c|c|c|}
\hline Peptide no. ${ }^{\text {a }}$ & Start position & Subsequence $^{\mathrm{b}}$ & BIMAS score ${ }^{c}$ & $\mathrm{RA}^{\mathrm{d}}$ & $\mathrm{DC}_{50}(\mathrm{~h})^{\mathrm{e}}$ \\
\hline 1 & 540 & ILAKFLHWL & 1745.714 & 2 & 8 \\
\hline 2 & & ILAKFLHW $\underline{\mathrm{V}}$ & 5683.72 & 2 & $>8$ \\
\hline 3 & 572 & RLFFYRKSV & 257.342 & 32 & $<2$ \\
\hline 4 & & YLFFYRKSV & 1183.775 & 4 & 4 \\
\hline 5 & 943 & LLQAYRFHA & 48.984 & 8 & 4 \\
\hline 6 & & LLQAYRFHE & 685.783 & 4 & $>8$ \\
\hline 7 & 926 & LQVNSLQTV & 27.573 & 4 & $>8$ \\
\hline 8 & & LLVNSLQTV & 271.948 & 2 & $>8$ \\
\hline 9 & 579 & SVWSKLQSI & 17.427 & 32 & 2 \\
\hline 10 & & SLWSKLQSI & 199.162 & 8 & $>8$ \\
\hline 11 & 945 & QAYRFHACV & 11.747 & 8 & 4 \\
\hline 12 & & QLYRFHACV & 845.752 & 4 & 6 \\
\hline 13 & 944 & LQAYRFHAC & 1.627 & 32 & 4 \\
\hline 14 & & LQAYRFHAV & 22.777 & 2 & $>8$ \\
\hline 15 & 23 & PLATFVRRL & 0.822 & $>32$ & 4 \\
\hline 16 & & $\underline{\text { YLATFVRRL }}$ & 171.771 & 4 & $>8$ \\
\hline 17 & 847 & GDMENKLFA & 0.576 & $>32$ & $<2$ \\
\hline 18 & & GLMENKLFA & 414.505 & $>32$ & $>8$ \\
\hline 19 & 925 & DLQVNSLQT & 0.559 & $>32$ & $<2$ \\
\hline 20 & & YLQVNSLQT & 34.279 & $>32$ & $<2$ \\
\hline
\end{tabular}

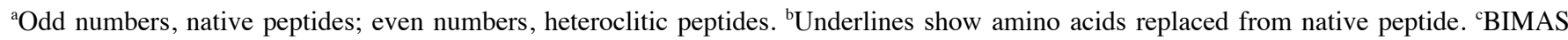
score was used for prediction of HLA-A*0201-binding peptides. ${ }^{\mathrm{d}} \mathrm{RA}$ was determined as described in Materials and methods. Lower RA value shows higher binding affinity for HLA-A2.1. ${ }^{\mathrm{e}} \mathrm{DC}_{50}$ was defined as the time required for the loss of $50 \%$ of the HLA-A2.1/peptide complexes stabilized at $\mathrm{t}=0$. $\mathrm{RA}$, relative affinity.

liposome solution was passed through a membrane filter with a pore size of $0.2 \mathrm{~mm}$.

Coupling of peptide to liposomes. Liposomal conjugates with TERT peptides were prepared essentially in the same manner as previously described (6). The final preparations contained $\leq 0.7 \mathrm{mg}$ of the peptide and $10 \mathrm{mg}$ of the liposome $/ \mathrm{ml}$ (24).

Immunization. The mice were immunized with indicated doses of liposomal peptides via subcutaneous injection in the presence of $5 \mu \mathrm{g} / \mathrm{mouse} \mathrm{CpG}$ or $100 \mu \mathrm{g}$ of peptide emulsified in $250 \mu \mathrm{l}$ of IFA with $100 \mu \mathrm{g}$ of peptide HBV core 128-140. These immunizations were performed on day 0 and 7 .

Intracellular IFN- $\gamma$ and CD107a degranulation analysis. After 5 days following immunization, spleen cells of immunized mice were incubated with brefeldin A (GolgiPlug; BD Biosciences) and fluorescein isothiocyanate (FITC)-conjugated monoclonal antibody (mAb) to CD107a for $5 \mathrm{~h}$ at $37^{\circ} \mathrm{C}$ in the presence or absence of a relevant peptide. After the incubation, the cells were washed, labeled with peridinin chlorophyll protein complex (PerCP)-conjugated anti-CD8 $\alpha \mathrm{mAb}$, fixed and permeabilized (Cytofix-Cytoperm; BD Biosciences) before they were labeled with phycoerythrin (PE)-conjugated anti-IFN- $\gamma$ mAb. All the antibodies were purchased from Biolegend. The stained cells were analyzed on a FACSCant II flow cytometer (BD Biosciences).
HLA-A2.1 dextramer analysis. To quantify antigen-specific $\mathrm{T}$ cells, the spleen cells were incubated with allophycocyanin (APC)-conjugated HLA dextramer recognizing 944v peptide derived from hTERT (944v/dextramer; Immudex, Copenhagen, Denmark) for $20 \mathrm{~min}$ at room temperature. After the incubation, the cells were washed, labeled with PerCP-conjugated anti-CD $8 \alpha \mathrm{mAb}$. The stained cells were analyzed on a FACSCant II flow cytometer (BD Biosciences).

CTL generation and cytotoxic assay. After 5 days following immunization, the spleen cells of immunized mice were cultured for 6 days with irradiated (30 Gy), syngeneic naïve spleen cells pre-pulsed with a relevant peptide and employed as effector cells in CTL-mediated cytotoxic assay. TERT-positive RMA-HHD cells were used as target cells. CTL-mediated cytotoxic assay was performed using a LIVE/DEAD Cell-mediated Cytotoxicity Assay kit (Life Technologies, Carlsbad, CA, USA) according to the manufacturer's instructions.

In vivo tumor protection. The HHD mice were vaccinated with liposomal peptide with $5 \mu \mathrm{g}$ of $\mathrm{CpG}$ on day 14,7 and 0 . On day 0 , the mice were challenged subcutaneously with $2 \times 10^{4}$ RMA-HHD cells. Tumor size was monitored every 3 to 4 days using calipers and the mice were sacrificed when the tumor mass reached $>1,000 \mathrm{~mm}^{2}$ or tumor tissue disintegration occurred. 
Table II. Contents of peptide groups.

\begin{tabular}{lc}
\hline Peptide groups & Peptide no. \\
\hline 1 & $1,5,9,13,17$ \\
2 & $3,7,11,15,19$ \\
3 & $2,6,10,14,18$ \\
4 & $4,8,12,16,20$ \\
\hline
\end{tabular}

Statistical analysis. Data were presented as the mean \pm SD. Statistical comparison between groups was based on the Mann-Whitney U test using Microsoft Excel. Mouse survival time was estimated using the Kaplan-Meier method and the Log-rank test. $\mathrm{P}<0.05$ was considered significant.

\section{Results}

Selection of CTL epitopes derived from hTERT for coupling to liposomes. Ten native peptides derived from hTERT were selected by the BIMAS score (odd numbers in Table I). To improve the binding affinity for HLA-A2.1, these peptides were subjected to amino acid substitutions as shown in Table I (even numbers). Most of the heteroclitical peptides improved their RA and $\mathrm{DC}_{50}$ value compared with the native peptides (Table I). To assess the immunogenicity, these peptides were pooled into four groups consisting of five peptides (Table II) and coupled to the surface of liposomes.
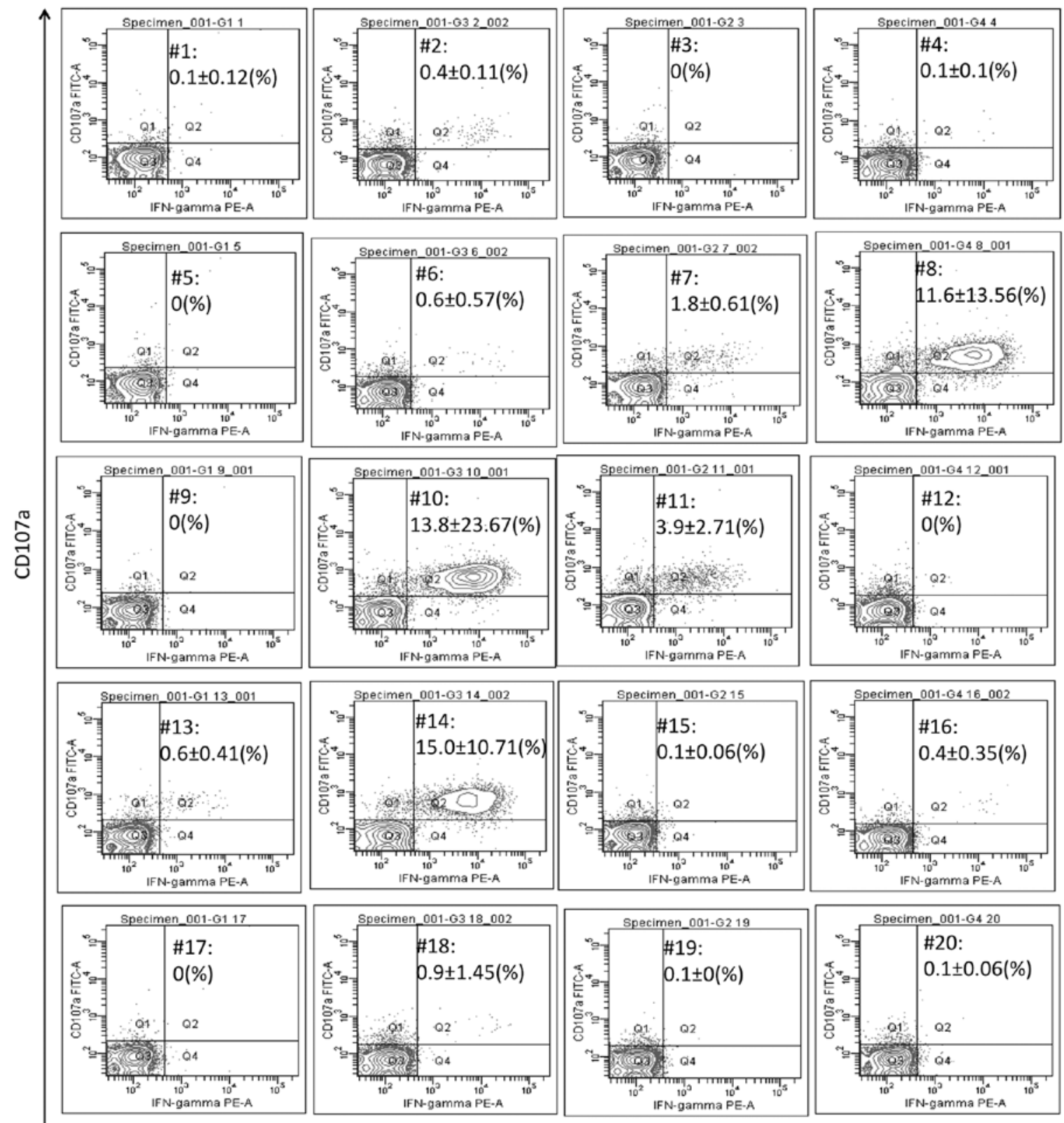

IFN-Y

Figure 1. Immunogenicity of predicted liposomal peptides. Spleen cells from HHD mice vaccinated with a peptide pool conjugated to liposomes were restimulated in vitro with a relevant peptide for $5 \mathrm{~h}$. CD8 T cells were tested for reactivity to each peptide by flow cytometric assay using the degranulation of CD107a and intracellular IFN- $\gamma$ staining. Percentages of IFN- $\gamma^{+}$CD107a cells in CD8 ${ }^{+}$spleen cells are presented as the mean \pm SD of three mice. 

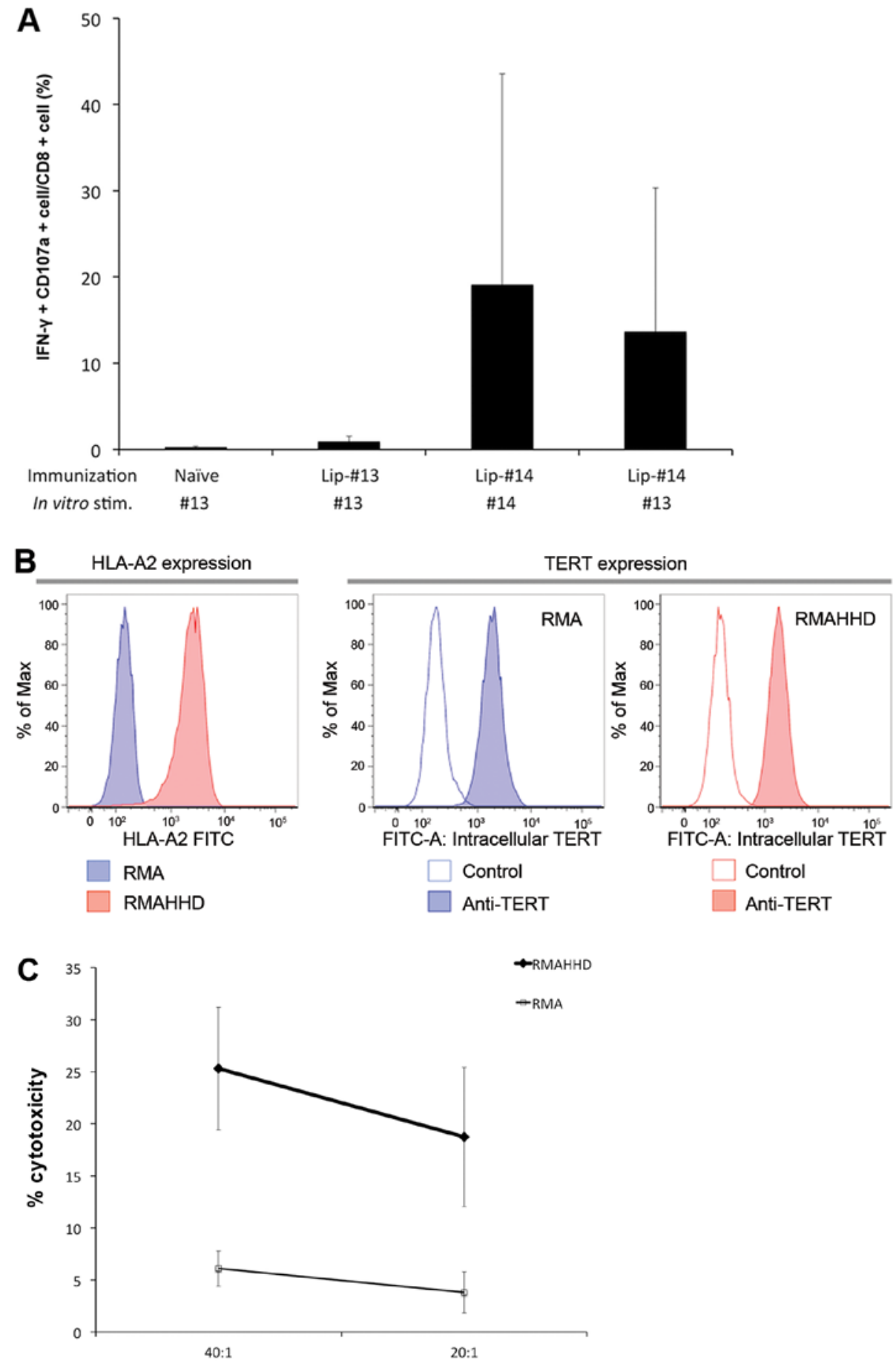

Figure 2. Generation of native cryptic epitope reactive CD8 T cells by vaccination with liposomal heteroclitic valiant. (A) CD8 T cells from Lip-\#14 immunized mice recognized \#14 as well as native epitope \#13. Spleen cells from the HHD mice vaccinated with Lip-\#13 (native) or Lip-\#14 (heteroclitic) were restimulated in vitro with \#13 or \#14 peptide for $5 \mathrm{~h}$. CD8 T cells were tested for specificity of each peptide by flow cytometric assay using the degranulation of CD107a and intracellular IFN- $\gamma$ staining. Results are presented as the mean \pm SD of three mice. (B) Flow cytometric analysis of HLA-2.1 and TERT expression on RMA and RMA-HHD mouse lymphoma cell lines. (C) CTL activities against TERT-expressing tumor cells. Spleen cells from the HHD mice vaccinated with Lip-\#14 were restimulated in vitro with \#14 peptide. After 6 days, the CTL-mediated cytotoxic assay was performed with TERT-expressing RMA and RMA-HHD cells as the target.

Each liposomal peptide pool was subsequently injected into the HHD mice. After 5 days following immunization, the spleen cells of immunized mice were prepared, stimulated with a relevant peptide and stained for their expression of surface CD8 and intracellular IFN- $\gamma$ and degranulation of CD107a. As shown in Fig. 1, three heteroclitical peptides on the surface of liposomes (Lip-\#8, -\#10 and-\#14) were able to induce a significant amount of IFN- $\gamma^{+} \mathrm{CD} 107 \mathrm{a}^{+} \mathrm{CD} 8^{+} \mathrm{T}$ cells and the responses were much higher compared to those induced by the original peptides (Lip-\#7, -\#9 and-\#13). Lip-\#14 was most effective for the induction of peptide-specific IFN- $\gamma^{+} \mathrm{CD} 107 \mathrm{a}^{+} \mathrm{CD} 8^{+} \mathrm{T}$ cells. Thus, \#14 (p944v), the heteroclitical peptide derived from \#13 (p944), was selected as a candidate epitope. 

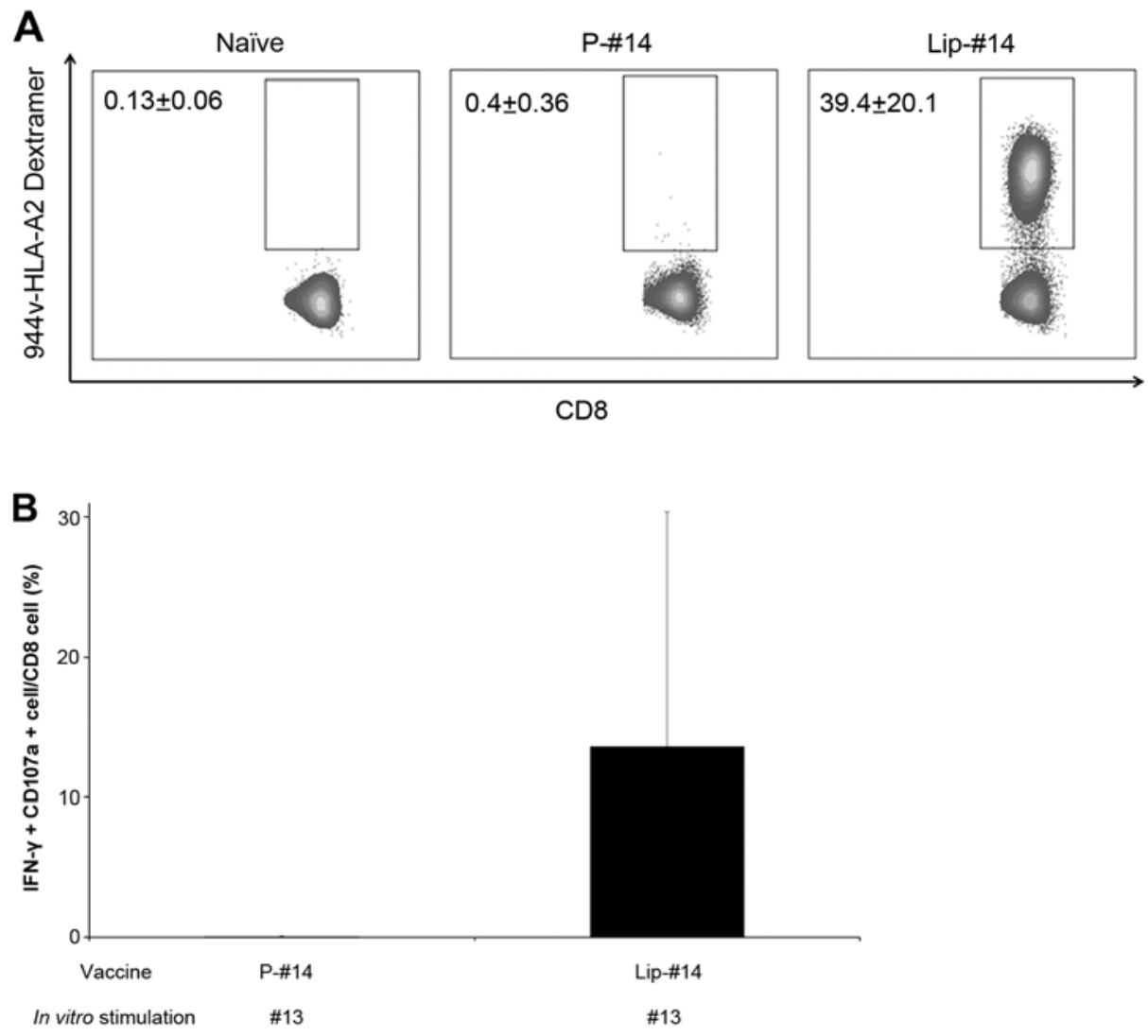

Figure 3. Comparison of native cryptic epitope specific CD8 T-cell responses in mice immunized with \#14 peptide conjugated liposomes or with \#14 peptide emulsified in IFA. (A) Spleen cells from naïve HHD or HHD mice vaccinated with Lip-\#14 or with P-\#14 were stained with \#14/HLA-A2.1-dextramer. Representative FACS plot of dextramer staining and the mean \pm SD of dextramer ${ }^{+} \mathrm{CD}^{+}$cells of three mice are presented. (B) Spleen cells from HHD mice vaccinated with Lip-\#14 or with P-\#14 were restimulated in vitro with \#13 peptide for $5 \mathrm{~h}$. CD8 T cells were tested for specificity of the peptide by flow cytometric assay using the degranulation of CD107a and intracellular IFN- $\gamma$ staining.

The heteroclitical peptide coupled to liposome (Lip-\#14) induces a strong CD8 T-cell response with cross recognition to the native epitope and in vivo primed CTLs with Lip-\#14 recognizes endogenously mTERT-expressing tumor cells.

The \#13 peptide (p944) and \#14 peptide (p944v) were, respectively, coupled to the surface of liposomes (Lip-\#13 and -\#14). These liposomal peptides were subsequently injected into the HHD mice and an intracellular IFN- $\gamma$ and CD107a degranulation analysis was conducted to assess antigen-specific CD8 T-cell responses against the native epitope \#13 (p944). As shown in Fig. 2A, Lip-\#14 induced strong CD8 T-cell responses in vivo. Compared with immunization with liposomal native \#13 peptide, the magnitude of CD8 responses induced by liposomal \#14 peptide was extremely strong. The results also showed that CD8 $\mathrm{T}$ cells that derived from Lip-\#14-immunized mice crossreacted with the native epitope $\# 13$ equally to the heteroclitic epitope \#14.

Additionally, to evaluate whether \#13 peptide is naturally processed and presented by tumor cells,\#14-specific CTLs were tested for their ability to lyse endogenously mTERT-expressing HLA-A2.1 $1^{+}$tumor cells. CTLs specific for \#14 were generated from the spleen cells of mice immunized with Lip-\#14 and their cytolytic activity was tested against RMA-HHD mouse lymphoma cells. Fig. 2B shows that RMA-HHD cells express mTERT endogenously while also possessing sufficient cell surface HLA-A2.1. We found that CTLs specific for \#14 were able to lyse RMA-HHD cells, but no cytotoxicity was observed against HLA-A2.1-negative RMA cells (Fig. 2C). These results indicated that \#13, the low affinity cryptic epitope, is naturally processed and presented by HLA-A2.1 molecules on the surface of TERT-expressing tumor cells and CTLs primed in vivo with Lip-\#14 immunization are able to recognize and kill tumor cells in an HLA-A2.1-restricted manner.

Liposomal \#14 peptide induces a stronger antigen-specific CD8 T-cell response than peptide + IFA immunization. Two vaccines were produced: Lip-\#14 and a \#14 peptide emulsified in IFA with a MHC class II pan T-helper peptide, HBVcore128-140 (24) (P-\#14). The immunogenicity of these two forms of TERT 944v was compared by analyzing spleen cells from the immunized mice using a TERT 944v-HLA-A2 dextramer assay (Fig. 3A), intracellular IFN- $\gamma$ and CD107a degranulation analysis (Fig. 3B). We found that an immunization with Lip-\#14 induced greater antigen-specific CD8 T cell response compared with the $\mathrm{P}-\# 14$ vaccination. The frequencies of TERT 944v-HLA-A2 dextramer ${ }^{+}$CD8 T cells detected in HHD mice were $~ 100$-fold higher after Lip-\#14 vaccination, compared to $\mathrm{P}$ - $\# 14$ vaccination $(39.4 \pm 20.1$ vs. $0.4 \pm 0.36 \%)$. As dextramer analysis did not discriminate between anergic and functional $\mathrm{T}$ cells, we also monitored an antigen-specific response by intracellular IFN- $\gamma$ and CD107a degranulation analysis. In agreement with results from the dextramer analysis, the frequencies of intracellular IFN- $\gamma$ and $\mathrm{CD} 107 \mathrm{a}^{+}$ CD8 T cells in response to native the \#13 epitope detected 

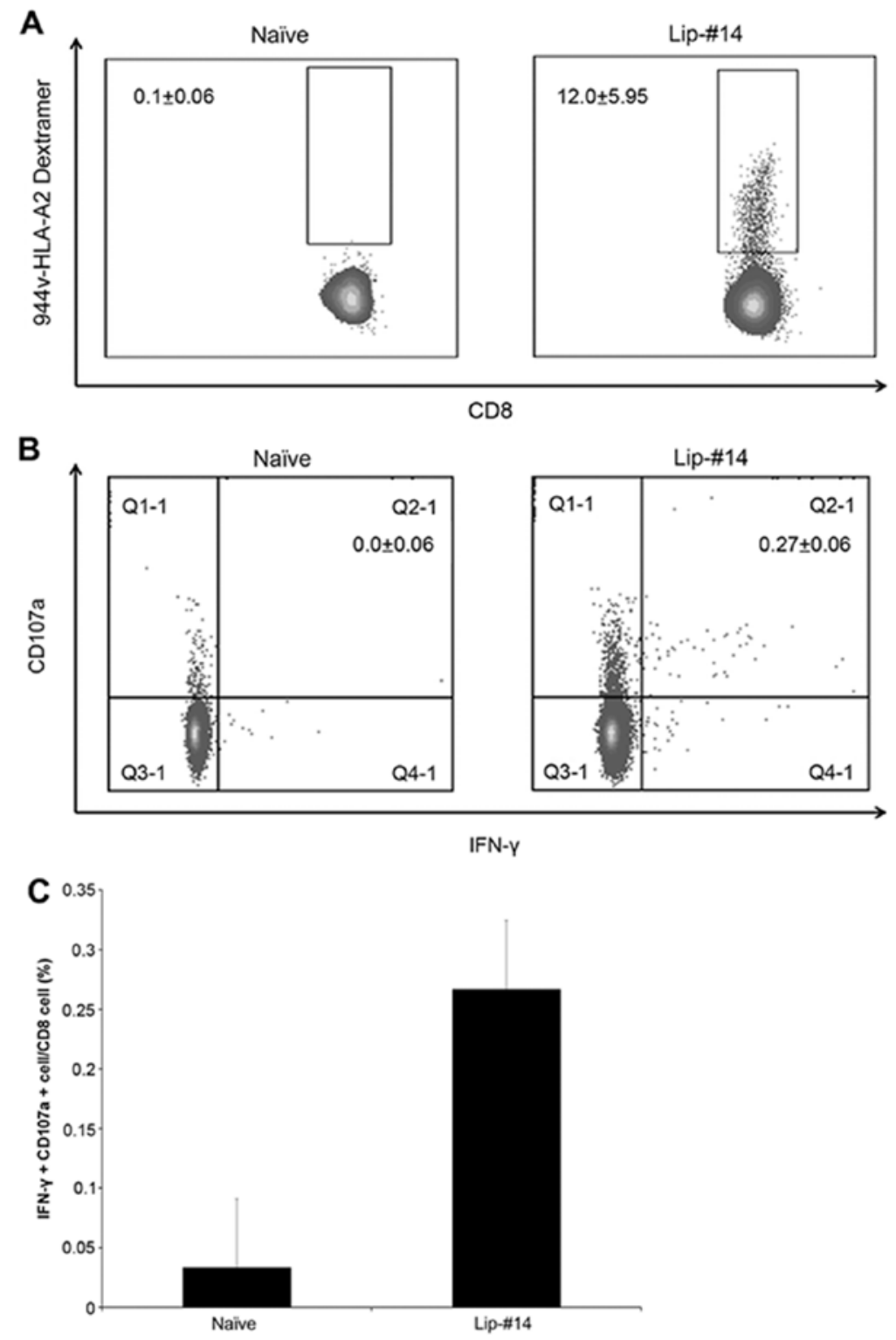

Figure 4. Induction of long-lasting memory CD8 T cells. (A) Presence of long-lasting antigen-specific CD8 T cells. HHD Mice were immunized with Lip-\#14. On day 35 after immunization, spleen cells were stained with \#14/HLA-A2.1-dextramer. Representative FACS plot of dextramer staining and the mean \pm SD of dextramer ${ }^{+} \mathrm{CD}^{+}$cells of three mice are presented. (B and C) Immune responses of long-lasting antigen-specific CD8 $\mathrm{T}$ cells. The abovementioned spleen cells were restimulated in vitro with \#13 peptide for $5 \mathrm{~h}$. The CD8 T cells were tested for specificity of the peptide by flow cytometric assay using the degranulation of CD107a and intracellular IFN- $\gamma$ staining.

in Lip-\#14-vaccined HHD mice were in contrast with the P-\#14-vaccined group (Fig. 3B). These results demonstrated that vaccination with surface-coupled liposomal antigen, which was modified to enhance their affinity for class I molecules causes strong immune responses against an epitope that cannot induce immune responses by ordinary vaccination such as peptide with IFA.

Previous reports suggest that the efficacy of active immunotherapy may be associated with the capacity of the vaccine to promote sustained CD8 T-cell responses in vivo (25). Therefore, we investigated whether long-lasting \#14-specific and \#13-crossreactive CD8 T cells could be elicited in HHD mice immunized with Lip-\#14. After 35 days following immunization, the CD8 responses were measured using a dextramer assay and flow cytometric analysis using intracellular IFN- $\gamma$ and CD107a degranulation. As shown in
Fig. 4A, TERT \#14-specific CD8 T cells were detected in mice on day 35 after immunization with Lip-\#14. When the long-lasting CD8 T-cell responses were evaluated by intracellular IFN- $\gamma$ and CD107a degranulation assay, we found that significant IFN $\gamma^{+} \mathrm{CD} 107 \mathrm{a}^{+} \mathrm{CD} 8 \mathrm{~T}$ cells in response to native \#13 peptide can only be detected in HHD mice injected with Lip-\#14 (Fig. 4B and C). These results suggested that immunization with Lip-\#14 is capable of effectively generating long-lasting memory CD8 T cell and sustained CD8 T-cell responses against native cryptic epitope.

Immunization with Lip-\#14 induces tumor growth inhibition. To investigate the antitumor effect of Lip-\#14, we immunized HHD mice with Lip-\#13 or -\#14 on day 14, 7 and 0. HHD mice immunized with irrelevant hepatitis $\mathrm{C}$ virus core 132-140 peptide coupled to the surface of liposomes were 

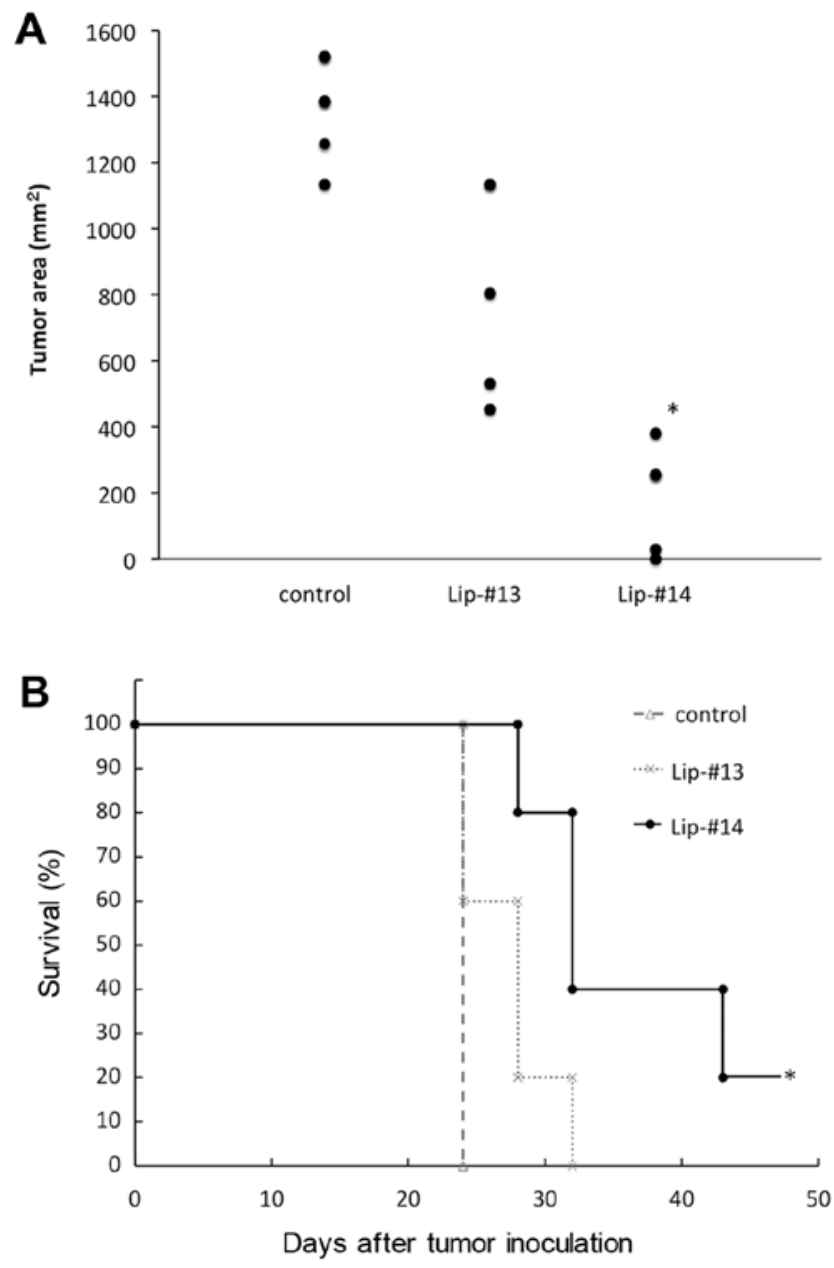

Figure 5. Immunization with liposomal heteroclitic variant inhibits tumor growth in vivo. HHD mice were immunized with control liposome, Lip-\#13 or -\#14 and inoculated with $2 \times 10^{4}$ RMA-HHD cells as indicated in Materials and methods. (A) Tumor sizes on day 24 after tumor inoculation. Tumor sizes in HHD mice vaccinated with Lip-\#14 were significantly differen from the control and Lip-\#13-vaccinated mice ( $\mathrm{P}<0.05$, Mann-Whitney U test). (B) Survival time of tumor inoculated mice. The survival time was estimated using the Kaplan-Meier method. "Statistically significant survival value between the Lip-\#14 group and the control liposome group $(\mathrm{P}<0.05$, Log-rank test $)$.

used as the negative control. On day 0 , we challenged HHD mice with $2 \times 10^{4}$ RMA-HHD lymphoma cells and monitored tumor growth and survival. The results showed that on day 24 following the challenge there was no significant difference in tumor size between Lip-\#13 and the control (Fig. 5A). This result suggested that immunization with Lip-\#13 did not induce sufficient protective antitumor immunity. By contrast, tumor size in Lip-\#14 immunized mice was significantly lower than the control and Lip-\#13 immunized mice $(\mathrm{P}<0.05$, Mann-Whitney U test, Fig. 5A). Furthermore, Lip-\#14 immunization prolonged the survival time of tumor-bearing mice compared with the control mice $(\mathrm{P}<0.05$, Log-rank test Fig. 5B). There was no significant difference in survival time between Lip-\#13 and the control (Fig. 5B). Thus, these results showed that, interference with tumor growth of TERT-expressing RMA-HHD cells was provided by immunization with Lip-\#14.

\section{Discussion}

In the present study, we utilized the high homology between mTERT and hTERT (26), which enabled us to examine epitopes exhibiting high immunogenicity on the surface of liposomes and test their immunotherapeutic potency by immunizing HHD mice and challenging with RMA-HHD lymphoma cells. In addition, by including a heteroclitic variant to each epitope candidate, we identified a novel epitope \#13 derived from TERT with low affinity to HLA-A2.1 molecules. The \#14, the heteroclitic peptide of \#13, had a high affinity to HLA-A2.1 and when coupled to the surface of liposome, induced strong and long-lasting $\mathrm{CD}^{+} \mathrm{T}$ cell responses against both \#14 and the original epitope \#13. Those responses were found to provide protection against endogenously TERTexpressing tumor cells.

As detailed characterization of many tumor cell expression molecules that act as TAAs is now available $(27,28)$, immunotherapy has become an increasingly essential component of cancer therapies $(29,30)$. Active cancer immunotherapy such as a cancer vaccine is based on fundamental knowledge that TAAs are presented on MHC molecules for recognition by specific $\mathrm{T}$ cells. However, the heterogeneous expression of most of the characterized TAAs limits the broad applicability of cancer vaccines. TAAs that can be broadly applied to various types of cancer, such as universal TAA, have been limited. Criteria for universal TAA include: i) expression by the vast majority of cancers, ii) presence of peptides that bind to MHC molecules, iii) sufficient natural antigen processing by tumor cells that leads to the expression of antigen-derived peptides in the groove of $\mathrm{MHC}$, and iv) recognition by $\mathrm{T}$ cell repertoire in an MHC-restricted manner. Previous studies have shown TERT meets these criteria (31). From these attractive fortes, TERT has been used as target of cancer vaccine (32). However, similar to most TAA, TERT is a self-antigen that is expressed on normal tissues, including the thymus (33). This fact raises the issue of tolerance of the TERT-specific T-cell repertoire and consequently the inability to trigger a strong and efficient antitumor immune response (14). Previous reports have shown that, self antigen-specific tolerance influences mainly the T-cell repertoire against dominant self-epitopes and to a much lesser extent against the cryptic epitope (34-36). Thus, we examined the non-tolerized repertoire against low affinity epitopes of TERT as the target of the antitumor vaccination. Since low affinity epitopes may be non-immunogenic, we devised two strategies for enhancement of immunogenicity of the epitope. One was modification of the epitope sequence to enhance affinity to MHC molecules. The other was chemically coupling the epitope peptide to the surface of liposome.

It has been shown that immunization using heteroclitical peptides derived from low affinity TERT epitopes emulsified in IFA recruits non-tolerized CTL repertoire and are more efficient at inducing tumor immunity than high affinity epitopes (15). However, the \#14 peptide, the heteroclitical epitope peptide identified in the present study, induced only weak CD8 T-cell immune responses against the native epitope when emulsified in IFA and injected in HHD mice. By contrast, when coupled to the surface of liposomes, it induced strong CD8 T-cell immune responses against the native cryptic epitope \#13, while \#13 peptide on the surface of liposomes induced poor CD8 
T-cell responses. Therefore, we consider that the synergistic effect between heteroclitic modification and coupling to the surface of liposomes provided the strong immunogenicity of Lip-\#14 and induced strong CD8 T cell responses against native cryptic epitope. Moreover, \#1 peptide (540-548), which was reported to induce CD8 immunity against TERT when emulsified in IFA (11) had no immunogenicity when coupled to the surface of the liposomes. These findings suggest that coupling to the surface of liposomes affects immunogenicity of peptides. In a previous study, we showed that coupling to the surface of liposomes alters the immunogenicity of hepatitis $\mathrm{C}$ virus-derived peptides (24). The mechanisms underlying the alteration of immunogenicity of peptides by liposome coupling is unclear. We hypothesize that the difference of the immunogenicity is associated with the difference of antigen presentation mechanisms. Peptides emulsified in IFA are considered to fit into the pockets of MHC class I molecules on APCs replacing the original endogenously synthesized peptides and presented to CD8 T cells (37). On the other hand, we have shown that antigens chemically coupled to the surface of liposomes consisting of unsaturated fatty acid (oleoyl liposome) are cross-presented by APCs (4). Results from another study have shown that oleoyl liposomes may be taken up by APCs via at least two pathways: fusion with and penetration of plasma membranes and pinocytosis (38). These unique antigen presentation mechanisms may be responsible for the unique immunogenicity of the peptide antigens.

The efficient antitumor immune responses are often fraught with autoimmunity. Vitiligo-like depigmentation has been reported after immunotherapy against malignant melanoma (39). By contrast, adoptive transfer of p53-specific CTL provides p53-overexpressing tumor growth inhibition without autoimmunity (40). In the present study, we did not observe any adverse effect in liposomal antigeninjected animals despite evidence of the induction of strong TERT-specific CD8 T-cell responses. Previous findings have shown that there are differences in TERT expression in normal somatic and tumor tissues. Thus, TERT is a potential safe target for cancer immunotherapy (41). Gross et al showed that immunization with heteroclitic low affinity TERT peptide resulted in anti-TERT T-cell immunity without any adverse effect by their careful examination in various somatic tissues (15). Recent findings show the safety of immunotherapy against TERT are accumulating (15,41-43). However, future investigations should be conducted to address whether the telomerase inhibition by host immune system activated by immunotherapy can affect normal tissues.

In conclusion, vaccination with the liposome-coupled heteroclitical peptide derived from TERT elicits antitumor CD8 T-cell responses evading the influence of tolerance in HHD mice. Therefore, heteroclitical-modified antigens of cryptic epitope with low affinity for HLA molecules derived from tumor antigens coupled to the surface of liposome may play a role as an effective cancer vaccine candidate.

\section{Acknowledgements}

The present study was supported by Grant from the Ochiai memorial award 2011, the Saitama Medical University Internal Grant 2012 and the JSPS KAKENHI grant no.
25430156. The authors would like to thank Hiroe Akatsuka for his technical assistance, as well as Dr Masanori Matsui and Dr Nobuharu Kobayashi (Depertment of Microbiology, Saitama Medical University, Saitama, Japan) for their helpful discussions. We are also grateful to Dr F.A. Lemonnier (Pasteur Institute, Paris, France) for providing HHD mice and RMA-HHD cells.

\section{References}

1. Lee PP, Yee C, Savage PA, Fong L, Brockstedt D, Weber JS, Johnson D, Swetter S, Thompson J, Greenberg PD, et al: Characterization of circulating $\mathrm{T}$ cells specific for tumorassociated antigens in melanoma patients. Nat Med 5: 677-685 1999.

2. Kawakami Y, Eliyahu S, Delgado CH, Robbins PF, Sakaguchi K, Appella E, Yannelli JR, Adema GJ, Miki T and Rosenberg SA: Identification of a human melanoma antigen recognized by tumor-infiltrating lymphocytes associated with in vivo tumor rejection. Proc Natl Acad Sci USA 91: 6458-6462, 1994.

3. Peek LJ, Middaugh CR and Berkland C: Nanotechnology in vaccine delivery. Adv Drug Deliv Rev 60: 915-928, 2008.

4. Taneichi M, Ishida H, Kajino K, Ogasawara K, Tanaka Y, Kasai M, Mori M, Nishida M, Yamamura H, Mizuguchi J, et al: Antigen chemically coupled to the surface of liposomes are cross-presented to $\mathrm{CD} 8^{+} \mathrm{T}$ cells and induce potent antitumor immunity. J Immunol 177: 2324-2330, 2006.

5. Takagi A, Matsui M, Ohno S, Duan H, Moriya O, Kobayashi N, Oda H, Mori M, Kobayashi A, Taneichi M, et al: Highly efficient antiviral $\mathrm{CD}^{+} \mathrm{T}$-cell induction by peptides coupled to the surfaces of liposomes. Clin Vaccine Immunol 16: 1383-1392, 2009.

6. Taneichi M, Tanaka Y, Kakiuchi T and Uchida T: Liposomecoupled peptides induce long-lived memory CD8 T cells without CD4 T cells. PLoS One 5: e15091, 2010.

7. Vonderheide RH, Hahn WC, Schultze JL and Nadler LM: The telomerase catalytic subunit is a widely expressed tumorassociated antigen recognized by cytotoxic T lymphocytes. Immunity 10: 673-679, 1999.

8. Minev B, Hipp J, Firat H, Schmidt JD, Langlade-Demoyen P and Zanetti M: Cytotoxic T cell immunity against telomerase reverse transcriptase in humans. Proc Natl Acad Sci USA 97: 4796-4801, 2000.

9. Artandi SE and DePinho RA: Telomeres and telomerase in cancer. Carcinogenesis 31: 9-18, 2010.

10. Hanahan D and Weinberg RA: Hallmarks of cancer: The next generation. Cell 144: 646-674, 2011.

11. Liu JP, Chen W, Schwarer AP and Li H: Telomerase in cancer immunotherapy. Biochim Biophys Acta 1805: 35-42, 2010.

12. Arai J, Yasukawa M, Ohminami H, Kakimoto M, Hasegawa A and Fujita S: Identification of human telomerase reverse transcriptase-derived peptides that induce HLA-A24-restricted antileukemia cytotoxic T lymphocytes. Blood 97: 2903-2907, 2001.

13. Warnock MG and Goodacre JA: Cryptic T-cell epitopes and their role in the pathogenesis of autoimmune diseases. $\mathrm{Br} \mathrm{J}$ Rheumatol 36: 1144-1150, 1997.

14. Scardino A, Gross DA, Alves P, Schultze JL, Graff-Dubois S, Faure O, Tourdot S, Chouaib S, Nadler LM, Lemonnier FA, et al: HER-2/neu and hTERT cryptic epitopes as novel targets for broad spectrum tumor immunotherapy. J Immunol 168: 5900-5906, 2002.

15. Gross DA, Graff-Dubois S, Opolon P, Cornet S, Alves P, Bennaceur-Griscelli A, Faure O, Guillaume P, Firat H, Chouaib S, et al: High vaccination efficiency of low-affinity epitopes in antitumor immunotherapy. J Clin Invest 113: 425-433, 2004.

16. Tourdot S, Scardino A, Saloustrou E, Gross DA, Pascolo S, Cordopatis P, Lemonnier FA and Kosmatopoulos K: A general strategy to enhance immunogenicity of low-affinity HLA-A2. 1-associated peptides: Implication in the identification of cryptic tumor epitopes. Eur J Immunol 30: 3411-3421, 2000.

17. Thorn M, Wang M, Kløverpris H, Schmidt EGW, Fomsgaard A, Wenandy L, Berntsen A, Brunak S, Buus S and Claesson MH: Identification of a new hTERT-derived HLA-A*0201 restricted, naturally processed CTL epitope. Cancer Immunol Immunother 56: 1755-1763, 2007. 
18. Cole DK, Edwards ES, Wynn KK, Clement M, Miles JJ, Ladell K, Ekeruche J, Gostick E, Adams KJ, Skowera A, et al: Modification of MHC anchor residues generates heteroclitic peptides that alter TCR binding and T cell recognition. J Immunol 185: 2600-2610, 2010.

19. Pinolla-Ibarz J, May RJ, Koronitsvit T, Gomez M, Kappel B, Zakhaleva V, Zhang RH and Scheinberg DA: Improved human T-cell responses against synthetic HLA-0201 analog peptides derived from the WT1 oncoprotein. Leukemia 20: 2025-2033, 2006.

20. Parkhurst MR, Salgaller ML, Southwood S, Robbins PF, Sette A, Rosenberg SA and Kawakami Y: Improved induction of melanoma-reactive CTL with peptides from the melanoma antigen gp100 modified at HLA-A*0201-binding residues. J Immunol 157: 2539-2548, 1996.

21. Kubo RT, Sette A, Grey HM, Appella E, Sakaguchi K, Zhu NZ, Arnott D, Sherman N, Shabanowitz J, Michel H, et al: Definition of specific peptide motifs for four major HLA-A alleles. J Immunol 152: 3913-3924, 1994.

22. Pascolo S, Bervas N, Ure JM, Smith AG, Lemonnier FA and Pérarnau B: HLA-A2.1-restricted education and cytolytic activity of $\mathrm{CD} 8\left(^{+}\right) \mathrm{T}$ lymphocytes from beta 2 microglobulin (beta2m) HLA-A2.1 monochain transgenic $\mathrm{H}-2 \mathrm{Db}$ beta $2 \mathrm{~m}$ double knockout mice. J Exp Med 185: 2043-2051, 1997.

23. Adotévi O, Mollier K, Neuveut C, Dosset M, Ravel P Fridman WH, Tartour E, Charneau P, Wain-Hobson S and Langlade-Demoyen P: Targeting human telomerase reverse transcriptase with recombinant lentivector is highly effective to stimulate antitumor CD8 T-cell immunity in vivo. Blood 115 3025-3032, 2010.

24. Takagi A, Kobayashi N, Taneichi M, Uchida T and Akatsuka T: Coupling to the surface of liposomes alters the immunogenicity of hepatitis $\mathrm{C}$ virus-derived peptides and confers sterile immunity. Biochem Biophys Res Commun 430: 183-189, 2013.

25. Kaech SM, Wherry EJ and Ahmed R: Effector and memory T-cell differentiation: Implications for vaccine development. Nat Rev Immunol 2: 251-262, 2002.

26. Greenberg RA, Allsopp RC, Chin L, Morin GB and DePinho RA Expression of mouse telomerase reverse transcriptase during development, differentiation and proliferation. Oncogene 16 1723-1730, 1998

27. Forni G, Lollini PL, Musiani P and Colombo MP: Immunoprevention of cancer: Is the time ripe? Cancer Res 60: 2571-2575, 2000.

28. Spurrell EL and Lockley M: Adaptive immunity in cancer immunology and therapeutics. E Cancer Med Sci 8: 441, 2014.

29. Blattman JN and Greenberg PD: Cancer immunotherapy: A treatment for the masses. Science 305: 200-205, 2004.

30. Mellman I, Coukos G and Dranoff G: Cancer immunotherapy comes of age. Nature 480: 480-489, 2011.

31. Patel KP and Vonderheide RH: Telomerase as a tumor-associated antigen for cancer immunotherapy. Cytotechnology 45: 91-99, 2004
32. Beatty GL and Vonderheide RH: Telomerase as a universal tumor antigen for cancer vaccines. Expert Rev Vaccines 7: 881-887, 2008.

33. Liu K, Schoonmaker MM, Levine BL, June CH, Hodes RJ and Weng NP: Constitutive and regulated expression of telomerase reverse transcriptase (hTERT) in human lymphocytes. Proc Natl Acad Sci USA 96: 5147-5152, 1999.

34. Cibotti R, Kanellopoulos JM, Cabaniols JP, Halle-Panenko O, Kosmatopoulos K, Sercarz E and Kourilsky P: Tolerance to a self-protein involves its immunodominant but does not involve its subdominant determinants. Proc Natl Acad Sci USA 89: 416-420, 1992.

35. Nanda NK and Sercarz EE: Induction of anti-self-immunity to cure cancer. Cell 82: 13-17, 1995.

36. Uram JN, Black CM, Flynn E, Huang L, Armstrong TD and Jaffee EM: Nondominant CD8 T cells are active players in the vaccine-induced antitumor immune response. J Immunol 186: 3847-3857, 2011.

37. Townsend ARM, Rothbard J, Gotch FM, Bahadur G, Wraith D and McMichael AJ: The epitopes of influenza nucleoprotein recognized by cytotoxic T lymphocytes can be defined with short synthetic peptides. Cell 44: 959-968, 1986.

38. Tanaka Y, Taneichi M, Kasai M, Kakiuchi T and Uchida T: Liposome-coupled antigens are internalized by antigen-presenting cells via pinocytosis and cross-presented to CD8 T cells. PLoS One 5: e15225, 2010.

39. Teulings HE, Limpens J, Jansen SN, Zwinderman AH, Reitsma JB, Spuls PI and Luiten RM: Vitiligo-like depigmentation in patients with stage III-IV melanoma receiving immunotherapy and its association with survival: A systematic review and meta-analysis. J Clin Oncol 33: 773-781, 2015.

40. Vierboom MP, Nijman HW, Offringa R, van der Voort EI, van Hall T, van den Broek L, Fleuren GJ, Kenemans P, Kast WM and Melief CJ: Tumor eradication by wild-type p53-specific cytotoxic T lymphocytes. J Exp Med 186: 695-704, 1997.

41. Harley CB: Telomerase and cancer therapeutics. Nat Rev Cancer 8: 167-179, 2008.

42. Yan J, Pankhong P, Shin TH, Obeng-Adjei N, Morrow MP, Walters JN, Khan AS, Sardesai NY and Weiner DB: Highly optimized DNA vaccine targeting human telomerase reverse transcriptase stimulates potent antitumor immunity. Cancer Immunol Res 1: 179-189, 2013.

43. Kotsakis A, Papadimitraki E, Vetsika EK, Aggouraki D, Dermitzaki EK, Hatzidaki D, Kentepozidis N, Mavroudis D and Georgoulias V: A phase II trial evaluating the clinical and immunologic response of HLA-A $2\left(^{+}\right)$non-small cell lung cancer patients vaccinated with an hTERT cryptic peptide. Lung Cancer 86: 59-66, 2014. 